\title{
Doppler Broadening of In-Flight Positron Annihilation Radiation due to Electron Momentum
}

\section{Citation}

Hunt, A. W., D. B. Cassidy, P. A. Sterne, T. E. Cowan, R. H. Howell, K. G. Lynn, and J. A. Golovchenko. 2001. "Doppler Broadening of In-Flight Positron Annihilation Radiation Due to Electron Momentum." Physical Review Letters 86 (24) (June): 5612-5615. doi:10.1103/ physrevlett.86.5612.

\section{Published Version}

doi:10.1103/PhysRevLett.86.5612

\section{Permanent link}

http://nrs.harvard.edu/urn-3:HUL.InstRepos:21976371

\section{Terms of Use}

This article was downloaded from Harvard University's DASH repository, and is made available under the terms and conditions applicable to Other Posted Material, as set forth at http:// nrs.harvard.edu/urn-3:HUL.InstRepos:dash.current.terms-of-use\#LAA

\section{Share Your Story}

The Harvard community has made this article openly available.

Please share how this access benefits you. Submit a story.

\section{Accessibility}




\title{
Doppler Broadening of In-Flight Positron Annihilation Radiation due to Electron Momentum
}

\author{
A. W. Hunt, ${ }^{1}$ D. B. Cassidy, ${ }^{2, *}$ P. A. Sterne, ${ }^{3}$ T. E. Cowan, ${ }^{3, \dagger}$ R. H. Howell, ${ }^{3}$ K. G. Lynn, ${ }^{1,4}$ and J. A. Golovchenko ${ }^{2}$ \\ ${ }^{1}$ Center for Materials Research, Washington State University, Pullman, Washington 99164-2711 \\ ${ }^{2}$ Department of Physics, Harvard University, Cambridge, Massachusetts 02138 \\ ${ }^{3}$ Lawrence Livermore National Laboratory, Livermore, California 94551 \\ ${ }^{4}$ Department of Physics, Washington State University, Pullman, Washington 99164-2814
}

(Received 20 March 2001)

\begin{abstract}
We report the first observation of electron momentum contributions to the Doppler broadening of radiation produced by in-flight two-photon annihilation in solids. In these experiments an approximately $2.5 \mathrm{MeV}$ positron beam impinged on thin polyethylene, aluminum, and gold targets. Since energetic positrons easily penetrate the nuclear Coulomb potential and do not cause a strong charge polarization, the experimental annihilation line shapes agree well with calculations based on a simple independentparticle model. Moreover, annihilations with the deepest core electrons are greatly enhanced.
\end{abstract}

PACS numbers: 78.70.Bj, 41.75.Ht, 71.20.-b

Positron annihilation spectroscopy is a well-known technique by which the momentum spectra of electrons in a solid may be obtained [1-4]. After reaching thermal energies positrons diffuse through a solid, during which some positrons annihilate, while others become localized in open-volume defects and other trapping centers before annihilation [3]. For both the delocalized and trapped positrons, the dominant decay mode is by the emission of two $\gamma$ rays which are nearly collinear and have energies close to the electron rest mass energy of $m_{0} c^{2}$. Since a thermalized positron contributes a negligible amount of momentum to the annihilating pair, any deviations in the $\gamma$-ray energies from $m_{0} c^{2}=511 \mathrm{keV}$ can be directly correlated to the electron momentum [1,2]. Consequently, the width and shape of the $511 \mathrm{keV}$ annihilation line yields information concerning the electrons with which the positrons annihilate.

The inner-shell electrons in the vicinity of the atomic nuclei are characterized by high momenta and produce greater Doppler broadening compared to the low momenta outer-shell electrons. To reach these inner-shell electrons, the positrons must penetrate the repulsive potential of the nucleus and therefore the outer electrons are responsible for most of the annihilations. Moreover, the annihilation with these outer electrons is further enhanced because of the strong charge polarization induced by the positron [1]. This enhancement makes describing the annihilation rate with low-momenta electrons a complicated task, but for sufficiently high momenta positron-electron correlations become less important $[2,4]$. Theories neglecting these correlations are generally called independent-particle models (IPM) because the electron wave functions are assumed not to respond to the presence of the positron. Recently, Iwata et al. studied annihilations in a simpler isolated two-body positron-atom interaction by mixing noble gases with cold positrons stored in a modified Penning trap [5]. The results qualitatively matched an IPM based on a static Hartree-Fock approximation in both the low and high momentum regions of the spectra. How- ever, to obtain quantitative agreement, positron-electron correlations with the outer electrons had to be considered.

While positron-electron correlations are important at thermal energies, highly energetic positrons $(\sim \mathrm{MeV})$ do not polarize the target because the distance an electron traverses during the interaction is exceptionally small $\left(<1 \times 10^{-15} \mathrm{~m}\right)$ [6]. Hence an IPM should be completely applicable. Furthermore, the nuclear Coulomb potential is easily penetrated and all electrons annihilate with equal probability, making the IPM extremely simple. This penetration also increases annihilation with the deepest core electrons that cannot be observed using thermalized positrons. In this Letter, we report the first observation of Doppler broadening due to electron momentum in the $\gamma$ rays emitted from in-flight two-photon annihilation when highly energetic $(\sim 2.5 \mathrm{MeV})$ positrons traverse thin foils. The majority of previous in-flight positron annihilation studies have focused on quantitative cross section measurements for comparison with theoretical predictions [7-9]. While Doppler broadening due to the electrons was not measured, it was realized that this determined the ultimate resolution when searching for resonant phenomena in annihilation spectra [10]. In principal, the ability to measure this additional Doppler broadening can be developed into a new probe of electron momentum distributions similar to Compton or electron scattering techniques $[11,12]$.

As the momentum of the positron-electron center of mass increases, so too does the energy spread, $\Delta E$, of the two emitted photons (energy $E_{1}$ and $E_{2}$ ). This difference energy can be determined from energy-momentum conservation and can be written as

$$
\Delta E=E_{1}-E_{2}=\frac{\mathbf{c} \cdot \mathbf{p}-\frac{c^{2} \mathbf{p}^{2}}{E_{T}}}{1-\frac{\mathbf{c} \cdot \mathbf{p}}{E_{T}}} .
$$

Here, $E_{T}=E_{1}+E_{2}$ is the total energy of the annihilating system, $\mathbf{p}$ is the positron-electron momentum, and $\mathbf{c}$ is the velocity of light in the direction of $E_{1}$. This equation 
reduces to $\Delta E=\mathbf{c} \cdot \mathbf{p}$, for thermalized positron annihilation. For an energetic positron beam traversing a thin target, a good first approximation is to neglect the electron momentum and assume the positron momentum is fixed in the beam direction. Then Eq. (1) can be rearranged to

$$
E_{1}=\frac{m_{0} c^{2}}{1-\frac{c p_{+}}{E_{T}} \cos \theta},
$$

where $p_{+}$is the positron momentum and $\theta$ is the angle between the emitted photon and the beam direction. There is a one to one correspondence between the photon energy and its emission angle. For $2.65 \mathrm{MeV}$ positrons, the photon energies range from $276 \mathrm{keV}$ for $180^{\circ}$ emission to $3396 \mathrm{keV}$ for $0^{\circ}$ emission.

Although the Doppler shifts induced by energetic positrons are enormous, the momenta of the target electrons will further widen the $\gamma$-ray distribution, but to a much smaller extent. For example, aluminum $K$-shell electrons will produce an additional Doppler shift up to $20 \mathrm{keV}$. To observe these effects, thin targets with different electron momentum characteristics were fabricated and installed at the end of the $3 \mathrm{MeV}$ monoenergetic positron beam line at Lawrence Livermore National Laboratory. The targets were free standing foils of $60 \mu \mathrm{m}$ polyethylene, $24 \mu \mathrm{m}$ aluminum, and $3 \mu \mathrm{m}$ gold. Typical beam characteristics were $2 \times 10^{5} \mathrm{e}^{+} / \mathrm{s}$ in a $3 \mathrm{~mm}$ diameter spot, with a maximum angular divergence of $0.25^{\circ}$. The beam energy was $2.65 \mathrm{MeV}$ with an energy spread of less then $6 \mathrm{keV}$. The energy characteristics were measured by examining the position and width of the $K$-shell single photon annihilation line from the gold target. Since the vast majority of positrons did not annihilate in the targets, a $75^{\circ}$ bending magnet was placed further downstream and deflected any transmitted positrons to a well-shielded beam dump. This was a specially designed double focusing magnet with a $10^{\circ}$ vertical and horizontal acceptance angle. Multiple scattering measurements and calculations predicted an angular divergence of about half this value for the gold target, assuring efficient collection of the transmitted beam [13]. More details about the accelerator and beam line may be found elsewhere [13-15].

A multiple $\gamma$-ray spectroscopy system consisting of a $7.3 \mathrm{~cm}$ diameter high purity germanium (HPGe) detector and two $3^{\prime \prime} \mathrm{NaI}$ scintillators recorded the annihilation photons from the targets. The HPGe detector was placed in the forward direction $37.5 \mathrm{~cm}$ from the target and subtended a half angle of 5.6 . From Eq. (2), annihilation photons with energies in excess of $3300 \mathrm{keV}$ were constrained to emit in this forward cone. At these high energies, the detector resolution was $5.0 \mathrm{keV}$, as measured from the 2734 and $3218 \mathrm{keV}$ monoenergetic $\gamma$-ray lines of ${ }^{88} \mathrm{Y}$. The two $\mathrm{NaI}$ scintillators were placed on either side of the target chamber, $8.9 \mathrm{~cm}$ in front of the target, collecting photons emitted between $140^{\circ}$ and $163^{\circ}$. These detectors recorded the $\sim 300 \mathrm{keV}$ photons emitted in coincidence with the forward $\sim 3.37 \mathrm{MeV} \gamma$ rays from two-photon annihilation.
Low-background two-photon annihilation energy spectra were obtained by requiring a coincidence within $30 \mathrm{~ns}$ between the HPGe detector and one NaI scintillator.

Figure 1 shows the full $\gamma$-ray spectrum after $\sim 4.1 \times$ $10^{10}$ positrons traversed the $24 \mu \mathrm{m}$ aluminum target. A difficulty with this set of experiments was the large integration times needed; this spectra took over $47 \mathrm{~h}$ to collect with a coincident event rate of $\sim 2 \mathrm{~s}^{-1}$. The singles rate (i.e., count rate without the coincidence requirement) in the HPGe detector was $\sim 250 \mathrm{~s}^{-1}$, indicating that pulse pileup was negligible. The most intense peak is from thermalized positron annihilation at $511 \mathrm{keV}$ with 11000 integrated counts and is due to accidental coincidences. By removing the coincidence requirement, the $511 \mathrm{keV}$ yield increases by a factor of 750. The in-flight two-photon annihilation peak is seen at $3375 \mathrm{keV}$ with 6000 events. The FWHM of $27.4 \mathrm{keV}$ is over 5 times larger than the energy resolution of the HPGe detector and is primarily determined by the $\mathrm{NaI}$ detectors which identify the backward emitted $\gamma$ rays. Considering the angular acceptance of these detectors and Eq. (2), the expected width in an ideal experiment would be $27.6 \mathrm{keV}$. This ideal width narrows slightly when the real response of the $\mathrm{NaI}$ detectors is included. The Compton edge at $3135 \mathrm{keV}$ and the plateau at lower energy is due to $\gamma$ rays Compton scattering out of the HPGe detector. The smaller peaks at 2862 and $2351 \mathrm{keV}$ are the single and double $\gamma$-ray escape peaks from recombining positron-electron pairs created in the detector.

In order to highlight the Doppler broadening caused by the momentum of the target electrons, Fig. 2 expands the energy scale around the in-flight annihilation line of Fig. 1. If the electrons had zero momentum, the maximum detectable energy would be $E_{\max }=3390 \mathrm{keV}$. There is a distinct wing above this energy which we attribute to Doppler broadening of the forward annihilation $\gamma$ rays by the momentum of the $K$-shell electrons. This is shown

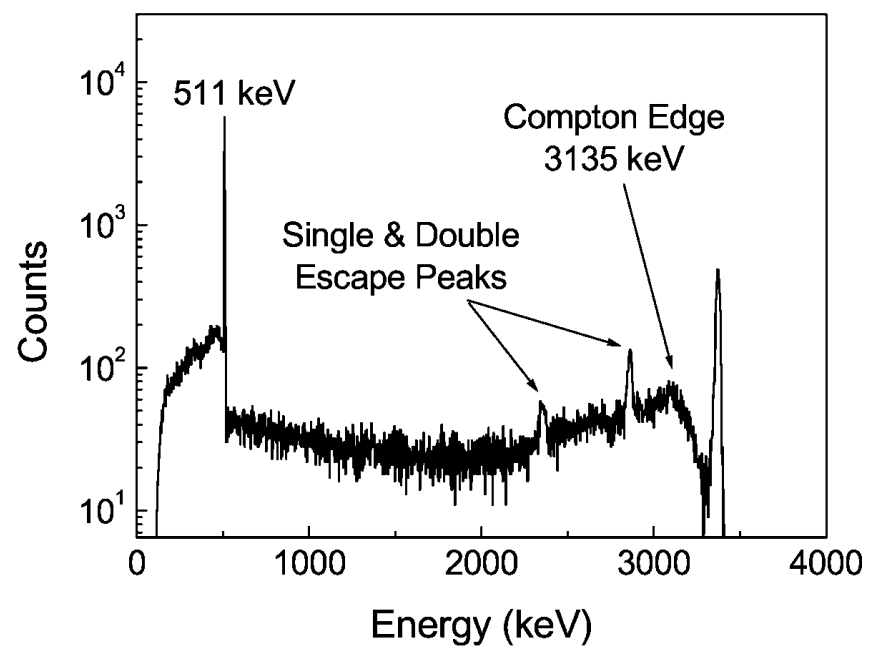

FIG. 1. Full $\gamma$-ray spectrum for the annihilation of $2.65 \mathrm{MeV}$ positron traversing $24 \mu \mathrm{m}$ of aluminum. This spectrum required a coincident photon in one of the NaI detectors within $30 \mathrm{~ns}$. The integration time was $41 \times 10^{9}$ positrons or 47 hours. 
by the theoretical curves also presented in Fig. 2. These curves are based on a very simple IPM in which all electrons are sampled equally. The calculations convolute the annihilation differential cross section, detection system response, positron energy spread, and the electron momentum distribution. The electron momentum distributions were calculated from a fully relativistic atomic structure calculation program using a local density form for the electron-electron interactions [16]. Although a complete electron momentum distribution was used, a much simpler model based on the virial theorem, $\left\langle\mathbf{p}^{2}\right\rangle=2 m\left|E_{B}\right|$, also gives good agreement with the experimental data $\left(E_{B}\right.$ refers to the binding energy of the electron).

The dotted curve in Fig. 2 uses an aluminum electron momentum distribution without the $K$ shell and it is clearly missing a significant fraction of the wing seen in the data. The dashed curve is the contribution from the aluminum $K$-shell electrons and it is apparent that these electrons are responsible for the wing at the highest energies. The $K$-shell contribution to the annihilation signal for energetic positrons in aluminum is $15 \%(2 / 13)$ which is 100 times larger than the $K$-shell contribution calculated for thermalized positron annihilation [17]. The solid line includes all electrons and a constant background has been added at low energies. This low energy background directly behind the peak is primarily due to incomplete charge collection in the HPGe detector, making it difficult to observe electron Doppler broadening effects in this region.

A dramatic demonstration of Doppler broadening from electron momentum is presented in Fig. 3 which compares

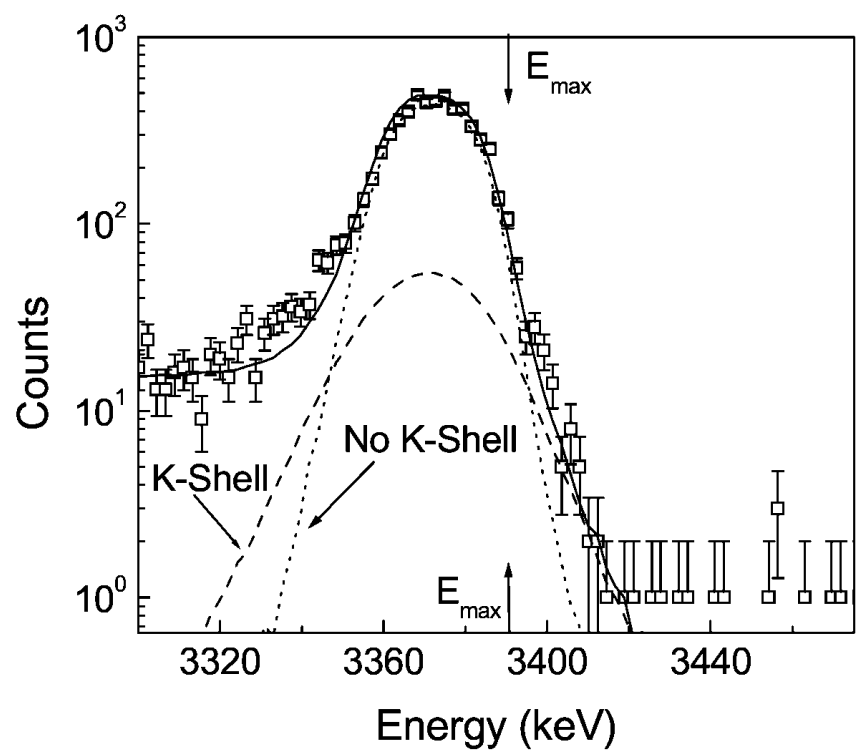

FIG. 2. Expanded energy scale around the in-flight annihilation peak at $3375 \mathrm{keV}$ in Fig. 1. The squares are the experimental aluminum spectrum with error bars of 1 standard deviation. The dotted curve is the calculated aluminum spectra without the $K$-shell electrons. The dashed curve is the $K$-shell contribution and the solid curve is the full aluminum calculation with a low energy background added. the annihilation spectra from the polyethylene and gold targets. The peak height has been normalized to unity and the energy scale on the horizontal axis has been shifted to $\delta E=E_{\gamma}-E_{\max }$. Only the high energy half of the peaks are plotted to easily distinguish the electron Doppler broadening effects. The deep core electrons of gold are tightly bound to the nucleus because of the large atomic number. These electrons and their associated high momenta produce large Doppler shifts leading to significant yields observed in the gold spectrum out to $\delta E=40 \mathrm{keV}$. In contrast, polyethylene $\left(\mathrm{CH}_{2}\right)$ lacks these high momenta electrons and hence the annihilation spectrum falls rapidly with no significant yield above $\delta E=13 \mathrm{keV}$. Moreover, the high energy wing in the aluminum spectrum caused by its $K$-shell electrons is absent in the polyethylene data.

Also shown in Fig. 3 is the calculated contribution from the $K$-, $L$-, and $M$-shell gold electrons which account for virtually all the annihilation yield above $\delta E=17 \mathrm{keV}$. This data constitute the first observation of Doppler broadening due to these deep core electrons in a high $Z$ material. In-flight two-photon annihilation with gold $M$-shell electrons has been detected for $70 \mathrm{keV}$ positrons by recording the energy of both annihilation $\gamma$ rays, $E_{T}=E_{1}+E_{2}$ [18]. Since energy is conserved the binding energy of the $M$-shell electrons shifted the centroid in this sum energy spectrum. However, Doppler broadening effects from the deep core electrons have not been observed using energetic or thermalized positrons. Typically with thermalized positrons annihilating in high $Z$ materials, only the shell immediately beneath the valence bands contributes significantly to the annihilation signal [5].

It is clear that the Doppler broadening due to electron momenta is observable here because energetic

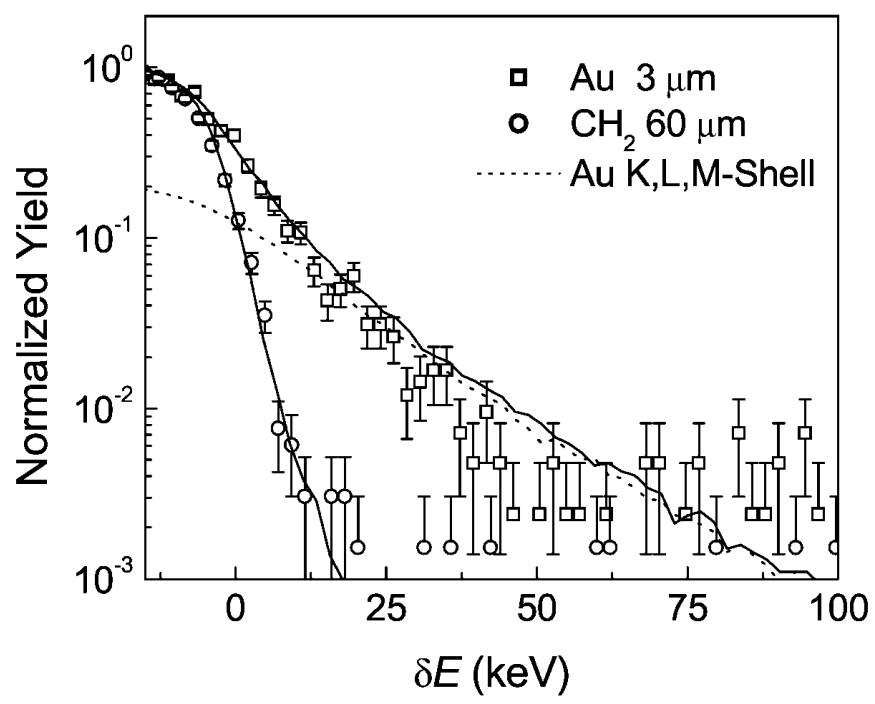

FIG. 3. Comparison of Doppler broadening produced by polyethylene $(\bigcirc)$ and gold $(\square)$ from $2.65 \mathrm{MeV}$ positrons. The solid lines are full calculations for the respective targets. The dotted curve is the $K, L$, and $M$-shell contribution for gold. The horizontal axis is plotted as $\delta E=E_{\gamma}-E_{\max }$, where $E_{\max }=$ $3390 \mathrm{keV}$ for our experimental conditions. 
positrons penetrate the Coulomb potential of the nucleus and annihilate with the deepest core electrons. These high momentum electrons are unobservable in standard positron annihilation spectroscopies because thermalized positrons are excluded from regions near the nuclei. The present experiment also contains annihilations from low momenta electrons, but is unable to resolve the associated Doppler shifts. In part this is due the relatively low flux, which renders data collection times impractical for higher resolution work. The inherent resolution can be estimated by adding in quadrature the energy resolution of the HPGe detector ( $5 \mathrm{keV}$ at $3.2 \mathrm{MeV})$ and the energy spread of the positron beam $(6 \mathrm{keV})$. This gives a momentum resolution of $4.2 p_{0}\left(p_{0}=1\right.$ a.u. of momentum), which is significantly larger than other positron annihilation or momentum spectroscopy techniques $[2,4,11,12,19]$. The true resolution is slightly more complicated because the annihilation line shape is not Gaussian and it is influenced by detector geometry.

Although the momentum resolution of the current experiment is modest, this is the first measurement to resolve the electron momentum using in-flight two-photon annihilation. The resolution can be drastically improved in a next generation of experiments which have improved positron flux. The Doppler shifts in the low energy $\sim 300 \mathrm{keV}$ coincident photons could be detected by replacing the NaI detectors with HPGe detectors. The energy resolution of these detectors at $\sim 300 \mathrm{keV}$ is typically better than $\sim 1 \mathrm{keV}$ which is a vast improvement over the resolution used here. Assuming a positron energy spread of $0.5 \mathrm{keV}$, the inherent momentum resolution would be $\sim 0.6 p_{0}$ which is comparable to standard 2detector positron annihilation spectroscopy [2,4]. However, to realize the benefits of this improved resolution it may be necessary to either deconvolute or reduce the energy spread caused by the angular acceptance of the $\gamma$ ray detectors. It may also be possible to measure the angular correlation of the annihilation radiation instead of the photon energies. In principal, the momentum resolution would be comparable to Compton and electron scattering techniques $[11,12]$. The technique presented here could then be combined with positron channeling. Channeled positrons are focused into the interstices of crystal, allowing the valence electron contribution to the annihilation yield to be enhanced by as much as $50 \%$ $[14,15]$. Moreover, the experimenter can control the core electron contribution by simply changing the incident angle of the positron beam. This may allow the electron momentum distribution to be spatially mapped within an atomic unit cell.
The authors would like to thank R. A. Levesque and J.C. Palathingal for helpful contributions to this research. This work was supported by the National Science Foundation under Contract No. DMR-9623610. This work was also performed under the auspices of the U.S. Department of Energy by the University of California, Lawrence Livermore National Laboratory under Contract No. W-7405-Eng-48.

*Present address: Department of Physics, Washington State University, Pullman, WA 99164-2814.

${ }^{\dagger}$ Present address: Photonics Division, General Atomics, San Diego, CA 92121-1122.

[1] R. A. Ferrell, Rev. Mod. Phys. 28, 308 (1956).

[2] K. G. Lynn, J. R. MacDonald, R. A. Boie, L. C. Feldman, J.D. Gabbe, M.F. Robbins, E. Bonderup, and J. Golovchenko, Phys. Rev. Lett. 38, 241 (1977)

[3] P. J. Schultz and K. G. Lynn, Rev. Mod. Phys. 60, 701 (1988)

[4] P. Asoka-Kumar, M. Alatalo, V. J. Ghosh, A. C. Kruseman, B. Nielsen, and K. G. Lynn, Phys. Rev. Lett. 77, 2097 (1996).

[5] K. Iwata, G. F. Gribakin, R. G. Greaves, and C. M. Surko, Phys. Rev. Lett. 79, 39 (1997).

[6] J.D. Jackson, Classical Electrodynamics (John Wiley \& Sons, New York, 1975).

[7] S. A. Colgate and F. C. Gilbert, Phys. Rev. 89, 790 (1953).

[8] J. B. Gerhart, B. C. Carlson, and R. Sherr, Phys. Rev. 94, 917 (1954).

[9] H. W. Kendall and M. Deutsch, Phys. Rev. 101, 20 (1956).

[10] W. H. Trzaska, H. Dejbakhsh, S. B. Dutta, Q. Li, and T. M. Cormier, Phys. Lett. B 269, 54 (1991).

[11] L. Dobrzyński, Z. Naturforsch A 48, 266 (1993).

[12] E. Weigold and I. McCarthy, Electron Momentum Spectroscopy (Kluwer Academic Publishers, Dordrecht, The Netherlands, 1999).

[13] F. A. Selim, Ph.D. thesis, Alexandria University, Egypt, 1999.

[14] A. W. Hunt, D. B. Cassidy, F. A. Selim, R. Haakenaasen, T.E. Cowan, R. H. Howell, K. G. Lynn, and J.A. Golovchenko, Nature (London) 402, 157 (1999).

[15] A. W. Hunt, D. B. Cassidy, F. A. Selim, R. Haakenaasen, T. E. Cowan, R.H. Howell, K. G. Lynn, and J.A. Golovchenko, Nucl. Instrum. Methods Phys. Res., Sect. B 164-165, 44 (2000).

[16] J. P. Desclaux, Comput. Phys. Commun. 1, 216 (1970).

[17] V. J. Ghosh, M. Alatalo, P. Asoka-Kumar, K. G. Lynn, and A. C. Kruseman, Appl. Surf. Sci. 116, 278 (1997).

[18] M.H. Weber, A.W. Hunt, J.A. Golovchenko, and K. G. Lynn, Phys. Rev. Lett. 83, 4658 (1999).

[19] P. E. Mijnarends, Physica (Amsterdam) 63, 248 (1973). 